\title{
Electrophysiological evidence of central interference in the control of visuospatial attention
}

\author{
Benoit Brisson and Pierre Joliceur \\ Université de Montréal, Montréal, Québec, Canada
}

\begin{abstract}
Visuospatial attention can be deployed to different locations in space without movement of the eyes. A large body of human electrophysiological studies reveals enhanced sensory-perceptual responses to stimuli that appear at an attended location. However, it is not clear that the mechanisms that underlie visuospatial attention are under the control of attention mechanisms that limit central processing in multiple-task situations. We investigated this question by incorporating a visual task that required the deployment of visuospatial attention as the second task of psychological refractory period (PRP) dual-task paradigms. The N2pc component of the event-related potential was used as an electrophysiological index of the moment-by-moment deployment of visuospatial attention to monitor when and where observers were attending while they performed concurrent central processing known to cause the PRP effect. Electrophysiological evidence shows that central processing interfered with the N2pc, suggesting that visuospatial attention is under the control of capacity-limited central mechanisms.
\end{abstract}

Visuospatial attention is known to improve performance when stimuli are presented at attended locations (Posner, 1980) and is also thought to be necessary to identify a predefined target in a search array, at least when difficult search tasks are performed (Treisman \& Gelade, 1980). Several electrophysiological studies (see Mangun, 1995, for a review) strongly suggest that early sensoryperceptual processing of attended stimuli is facilitated.

On the other hand, in multiple-task situations central attention selects information to be processed in capacitylimited central stages. The psychological refractory period (PRP) paradigm has been used extensively to study central attention. In the PRP paradigm, two distinct targets, $\mathrm{T}_{1}$ and $\mathrm{T}_{2}$, often presented in different sensory modalities, are separated by various stimulus onset asynchronies (SOAs), and a speeded response is required for each target. PRP interference effects are reflected in an increase in mean response time to the second target $\left(\mathrm{RT}_{2}\right)$ as the $\mathrm{SOA}$ is reduced (Pashler, 1994; Pashler \& Johnston, 1989). Manipulating response selection demands of $\mathrm{T}_{1}$ modulates the PRP effect (Karlin \& Kestenbaum, 1968), suggesting that response selection requires limited central attention mechanisms (Pashler, 1994; Pashler \& Johnston, 1989).

Although a large body of evidence shows that visuospatial attention enhances early sensory-perceptual stages of processing and central attention selects information to be processed at later, limited central stages, the relationship between these two types of attention is still unclear. Some researchers have claimed that visuospatial attention and central attention are independent (e.g., Johnston, McCann, \& Remington, 1995; Pashler, 1991), whereas others have claimed that they share common mechanisms (e.g., Jiang \& Chun, 2001).

Johnston et al. (1995) cleverly used the locus-of-slack logic to argue that visuospatial attention and central attention operate at different stages of processing. They nevertheless investigated these two types of attention independently in two separate experiments. This aspect of their study makes it difficult to observe possible interactions between visuospatial attention and central attention and, consequently, to determine whether or not they are independent. Furthermore, the opposite conclusions of Pashler (1991) and Jiang and Chun (2001) suggest that further work on the issue is warranted.

To bring new evidence to bear on this issue, we recorded event-related potentials (ERPs) in addition to behavioral measures. ERP components provide indexes of distinct covert stages of processing that occur between stimulus presentation and the overt response. The ERP component of interest in this study is called N2pc (Eimer, 1996; Luck \& Hillyard, 1994; Woodman \& Luck, 2003). The N2pc - an increased negativity at posterior electrode sites contralateral to the position of an attended visual target - typically occurs about 180-280 msec after target onset and likely indexes covert visuospatial attention in light of several results reviewed by Woodman and Luck (2003). We measured the N2pc elicited by a lateralized visual target under different concurrent central load conditions manipulated

B. Brisson, benoit.brisson@umontreal.ca 
using a modified PRP paradigm similar to that used by Pashler (1991).

If the control of visuospatial attention requires mechanisms or resources that overlap with those that control attention-limited central mechanisms, the interference of central attention (required for Task ${ }_{1}$ ) on visuospatial attention (required for $\mathrm{Task}_{2}$ ) should be reflected by an increase in N2pc latency and/or by a reduction in N2pc amplitude in a high-load condition relative to those in a low-load condition.

\section{EXPERIMENT 1}

In this experiment, $\mathrm{T}_{1}$ was a tone and $\mathrm{T}_{2}$ - which was embedded in a symmetric bilateral visual display - was a uniquely colored square with a gap on one side (see Figure 1). The subjects were required to make two speeded buttonpress responses on each trial, the first to indicate the pitch of $\mathrm{T}_{1}(200,430,926$, or $2000 \mathrm{~Hz})$ and the second to indicate the location of the gap in $\mathrm{T}_{2}$ (up, down, left, or right). Overlap between central processing of $T_{1}$ and deployment of visuospatial attention to $T_{2}$ was manipulated by varying the $\mathrm{T}_{1}-\mathrm{T}_{2}$ SOA. In the short-SOA condition, attention-limited central processing should still be engaged on $T_{1}$ when visuospatial attention must be deployed to $\mathrm{T}_{2}$. In contrast, in the long-SOA condition, the response to $\mathrm{T}_{1}$ should have usually been made by the time $\mathrm{T}_{2}$ is presented, and so capacity-limited central mechanisms should not be busy with $\mathrm{T}_{1}$. Thus, the long-SOA condition is a low-load control condition approximating single-task performance.

\section{Method}

Subjects. Eleven neurologically normal subjects participated in this experiment for financial compensation. Three were excluded from the analyses. Therefore, 8 subjects remained in the sample.

Materials and Procedure. Each subject performed one practice block of 64 trials followed by eight experimental blocks of 96 trials. The trial sequence is presented in Figure 1. Each trial was initiated by simultaneously pressing the "N" and "V" keys on a computer keyboard with the right and left index fingers, respectively. A fixation point appeared at the center of the computer screen, which was visible throughout the remainder of the trial. Five hundred millisec-

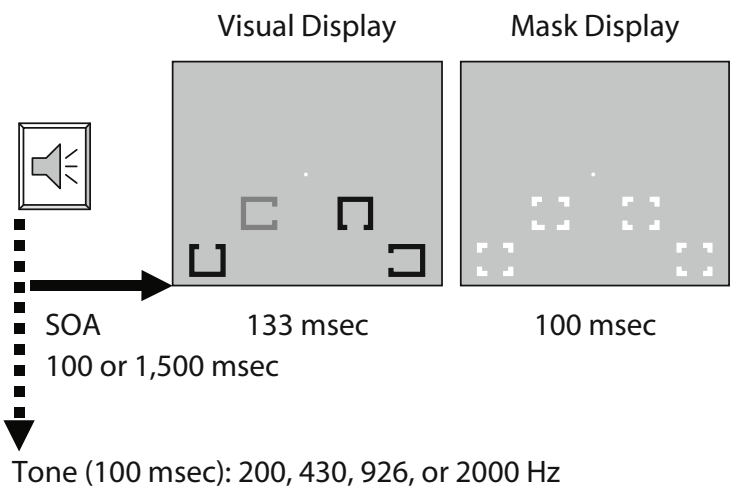

Figure 1. Stimulus sequence in Experiment 1. In the experiment, the stimuli were red and green in the visual display and gray in the mask display. onds later, a 100 -msec tone $\left(\mathrm{T}_{1}\right)$ was emitted by two speakers placed on each side of the computer screen. The tone was followed, at an SOA of 100 (short) or 1,500 (long) msec, by a 133-msec bilateral visual display that contained $\mathrm{T}_{2}$, which in turn was immediately followed by a $100-\mathrm{msec}$ bilateral mask. The visual display contained four colored squares (two on each side of fixation), each with a gap on one side (different for each square). The mask display consisted of four gray squares, each with gaps on all sides (see Figure 1). All squares in the visual and mask displays subtended a visual angle of $1^{\circ} \times 1^{\circ}$, and the gaps were $0.33^{\circ}$. The centers of the squares nearest to fixation were $1.5^{\circ}$ below and $3.5^{\circ}$ to the left or right of fixation. The centers of the far squares were $3^{\circ}$ below and $5^{\circ}$ to the left or right of fixation. $T_{2}$ appeared randomly on the left or right of fixation; it was red among green distractors for half of the subjects and green among red distractors for the other half. Both colors were approximately equiluminant to equalize their low-level sensory responses. Responses to $T_{1}$ were made with the fingers of the right hand (the response keys were those for the letters " $\mathrm{N}$ " and "M," the comma, and the period for the 200-, 430-, 926-, and 2000-Hz tones, respectively), and responses to $T_{2}$ were made with the fingers of the left hand (the response keys were those for the letters " $\mathrm{Z}$," "X," "C," and "V" for left, bottom, top, and right gaps, respectively).

The electroencephalogram (EEG) was recorded from 64 active Ag/ $\mathrm{AgCl}$ electrodes (BioSemi ActiveTwo system) mounted on an elastic cap and referenced to the average of the left and right mastoids. Electrodes were placed according to the International 10/10 system. The horizontal electrooculogram (HEOG), recorded as the voltage difference between electrodes placed lateral to the external canthi, was used to measure horizontal eye movements. The vertical electrooculogram (VEOG), recorded as the voltage difference between two electrodes placed above and below the left eye, was used to detect eye blinks. A bandpass filter of $0.01-67 \mathrm{~Hz}$ was applied, and the EEG and EOG signals, digitized at $256 \mathrm{~Hz}$, were averaged offline.

Trials with artifacts at electrode sites of interest $(\mathrm{O} 1, \mathrm{O} 2, \mathrm{PO} 7, \mathrm{PO} 8$, P7, and/or P8), eye blinks (VEOG $>100 \mu \mathrm{V}$ ), and large horizontal eye movements (HEOG $>35 \mu \mathrm{V}$ ) were excluded from the analysis.

Using the procedure described by Woodman and Luck (2003), 1 subject with residual eye movements that deviated more than $0.2^{\circ}$ (i.e., HEOG $>3.2 \mu \mathrm{V}$ ) toward the target after ocular artifact rejection was rejected from the analysis. Because we were investigating $\mathrm{N} 2 \mathrm{pc}$ modulations, we rejected 1 subject who did not elicit an N2pc in either condition (N2pc mean amplitude of less than $-0.3 \mu \mathrm{V}$ at PO7/PO8 sites in both conditions). ${ }^{1}$ One other subject was rejected because $\mathrm{T}_{2}$ accuracy was at chance.

\section{Results and Discussion}

Behavioral results are presented in Table 1. Only trials with correct responses to both $T_{1}$ and $T_{2}$ were included in the RT analyses, and outliers were excluded using the method described in Jolicœur (1999a). Repeated measures ANOVAs with SOA condition (short vs. long) as a factor revealed that $\mathrm{T}_{1}$ accuracy was not affected by $\mathrm{SOA}[F(1,7)=$ $1.1, p>.33] . \mathrm{RT}_{1}$ was slower at the short $\mathrm{SOA}[F(1,6)=$ $25.5, p<.003$ ] for 7 of the 8 subjects. ${ }^{2}$ Importantly, even though $\mathrm{Task}_{2}$ was identical in each SOA condition, behavioral results showed that $\mathrm{RT}_{2}$ was substantially longer in the short-SOA condition than in the long-SOA condition $[F(1,7)=587.9, p<.0001] .{ }^{3}$ Thus, the desired PRP effect was obtained. Furthermore, responses to $\mathrm{T}_{2}$ were less accurate in the short- than in the long-SOA condition $[F(1,7)=$ 29.1, $p<.001$ ], as Pashler (1991) also observed.

The increase in $\mathrm{RT}_{2}$ and the reduction in $\mathrm{T}_{2}$ accuracy as SOA was reduced suggests that central processing required for Task ${ }_{1}$ interfered with some aspects of $T_{2}$ processing. To assess more precisely where the interference occurred, we measured the N2pc component elicited by 
Table 1

Behavioral Results (and Standard Errors of the Means) for Experiments 1 and 2

\begin{tabular}{|c|c|c|c|c|c|c|c|c|c|}
\hline \multirow[b]{2}{*}{ Experiment } & \multirow[b]{2}{*}{ Condition } & \multicolumn{2}{|c|}{$\%$ Correct to $\mathrm{T}_{1}$} & \multicolumn{2}{|c|}{$\mathrm{RT}_{1}$} & \multicolumn{2}{|c|}{$\%$ Correct to $\mathrm{T}_{2}$} & \multicolumn{2}{|c|}{$\mathrm{RT}_{2}$} \\
\hline & & $M$ & $S E M$ & $M$ & SEM & $M$ & SEM & $M$ & $S E M$ \\
\hline 1 & Short SOA & 86 & 3 & 854 & 51 & 83 & 2 & 1,327 & 52 \\
\hline \multirow[t]{2}{*}{2} & $4 \mathrm{AD}$ & 85 & 3 & 870 & 109 & 96 & 1 & 1,162 & 76 \\
\hline & SRT & 100 & 0 & 288 & 47 & 94 & 2 & 462 & 21 \\
\hline
\end{tabular}

Note $-\mathrm{RT}_{1}$, response time (in milliseconds) to $\mathrm{T}_{1} ; \mathrm{RT}_{2}$, response time to $\mathrm{T}_{2}$. $\quad$ *See note 2 .

$\mathrm{T}_{2}$, which allowed us to monitor more directly the deployment of visuospatial attention. As we can observe in Figure $2 \mathrm{C}$, there are clearly no effects of load on N2pc latency. Therefore, we analyzed only N2pc amplitude.

The EEG was averaged starting $200 \mathrm{msec}$ prior to $\mathrm{T}_{2}$ onset and ending $500 \mathrm{msec}$ after $\mathrm{T}_{2}$ onset, and was baseline corrected on the basis of the 200-msec pretarget period. The ipsilateral waveform (average of left hemisphere with left visual field target and right hemisphere with right visual field target) and the contralateral waveform (average of left hemisphere with right visual field target and right hemisphere with left visual field target), time locked to $\mathrm{T}_{2}$ for the long-SOA condition, are displayed in Figure $2 \mathrm{~A}$, and those for the short-SOA condition are shown in Figure 2B. These waveforms are different across SOA conditions because the short-SOA condition in particular includes overlapping activity elicited by Task 1 . To isolate the N2pc from overlapping activity that was not lateralized with respect to the side of $\mathrm{T}_{2}$ (e.g., Task $_{1}$ stimulus, preparation, response activity), the N2pc was quantified as the average of the contralateral waveforms minus the average of the ipsilateral waveforms (Figure 2C). N2pc measurements (mean amplitude during the 180-260-msec postvisual display time window) were entered into an ANOVA with SOA condition (short vs. long) and electrode position $(\mathrm{O} 1 / \mathrm{O} 2$ vs. $\mathrm{PO} 7 / \mathrm{PO} 8$ vs. P7/P8) as factors. There was no interaction between electrode position and SOA. Therefore, we report in detail only the SOA effects, which were essential to the experimental design, at PO7/PO8 sites, where the N2pc was maximal.

The amplitude of the N2pc component was clearly reduced in the short-SOA condition relative to the longSOA condition $[F(1,7)=20.4, p<.003]$. The modulation of the N2pc mean amplitude in this experiment does not seem to be caused by jitter in the latency of the component ${ }^{4}$ and therefore strongly suggests that the subjects were not able to deploy their attention to $T_{2}$ as efficiently when central attention was engaged on Task $\mathrm{s}_{1}$. Since the SOA conditions were randomly intermixed in each block, the modulation of the N2pc could not be caused by a differential pretrial preparatory state. These results are therefore consistent with the hypothesis that central attention interferes with the control of visuospatial attention.

\section{EXPERIMENT 2}

The stimulus sequences in Experiment 1 were not exactly identical across central load because of the difference in SOA. ERPs can be sensitive to such differences, although it is unlikely that differences in the stimulus sequences would have produced the differences in the degree of lateralization of ERPs (e.g., N2pc). Nonetheless, a second experiment was conducted in which central load was manipulated by changing the nature of Task while SOA was held constant at 100 msec. Task $_{1}$ was either a (high-load) task requiring speeded four-alternative (4AD) discrimination of the pitch of the tone, which was identical to Task ${ }_{1}$ in Experiment 1, or a (low-load) task requiring a simple reaction time (SRT) response regardless of the tone. To generalize our results, a different visual display was used (see Figure 3) in which only two highly overlearned items (a letter and a digit) were presented, one on each side of fixation. The letter (A, B, C, or D) and the digit $(1,2,3$, or 4$)$ were in different equiluminant colors (one red and one green), and the subjects were required to make a speeded response to the identity of the item of a prespecified color.

\section{Method}

Subjects. Thirteen neurologically normal subjects participated in this experiment for financial compensation. Four were excluded for having no $\mathrm{N} 2 \mathrm{pc}$ (i.e., $<0.3 \mu \mathrm{V}$; see note 1 ) and 1 more was excluded for excessive average eye movements toward the target (i.e., $>.2^{\circ}$ of residual horizontal eye movement). Therefore, 8 subjects were included in the analyses.

Materials and Procedures. The stimuli and procedure were identical to those of Experiment 1, with the following exceptions. First, each trial was initiated by simultaneously pressing the "N" and " $\mathrm{X}$ " keys. Second, $\mathrm{T}_{1}-\mathrm{T}_{2}$ SOA was the same in both conditions. Third, in the SRT condition, the same speeded buttonpress response was required regardless of the tone. The $\mathrm{T}_{1}$ response key in the SRT condition was counterbalanced between subjects so that all four $\mathrm{T}_{1}$ response keys used in the 4AD condition (i.e., the keys for the letters " $\mathrm{N}$ " and "M," the comma, and the period) were also used in the SRT condition. Fourth, the $100-\mathrm{msec}$ visual display contained only one red and one green overlearned item (a digit and a letter) on each side of fixation, and the mask display contained two gray Ms (see Figure 3). Half of the subjects responded to the red item in the visual display, and the other half responded to the green item. Green and red items appeared randomly on the left or right of fixation. The red item was a letter for half of the subjects and a digit for the other half. All items subtended a visual angle of $1^{\circ}$ and were presented $3^{\circ}$ to the left or right of fixation. The letters A and B (or the digits 1 and 2) were mapped to the " $Z$ " key, and the letters $\mathrm{C}$ and $\mathrm{D}$ (or the digits 3 and 4) were mapped to the " $X$ " key.

\section{Results and Discussion}

Behavioral results are presented in Table 1. As was expected, $\mathrm{RT}_{1}$ was shorter in the SRT condition than in the $4 \mathrm{AD}$ condition $[F(1,7)=39.2, p<.001]$. Although Task 2 was identical in both conditions, mean $\mathrm{RT}_{2}$ was shorter in the SRT condition than in the $4 \mathrm{AD}$ condition $[F(1,7)=$ 

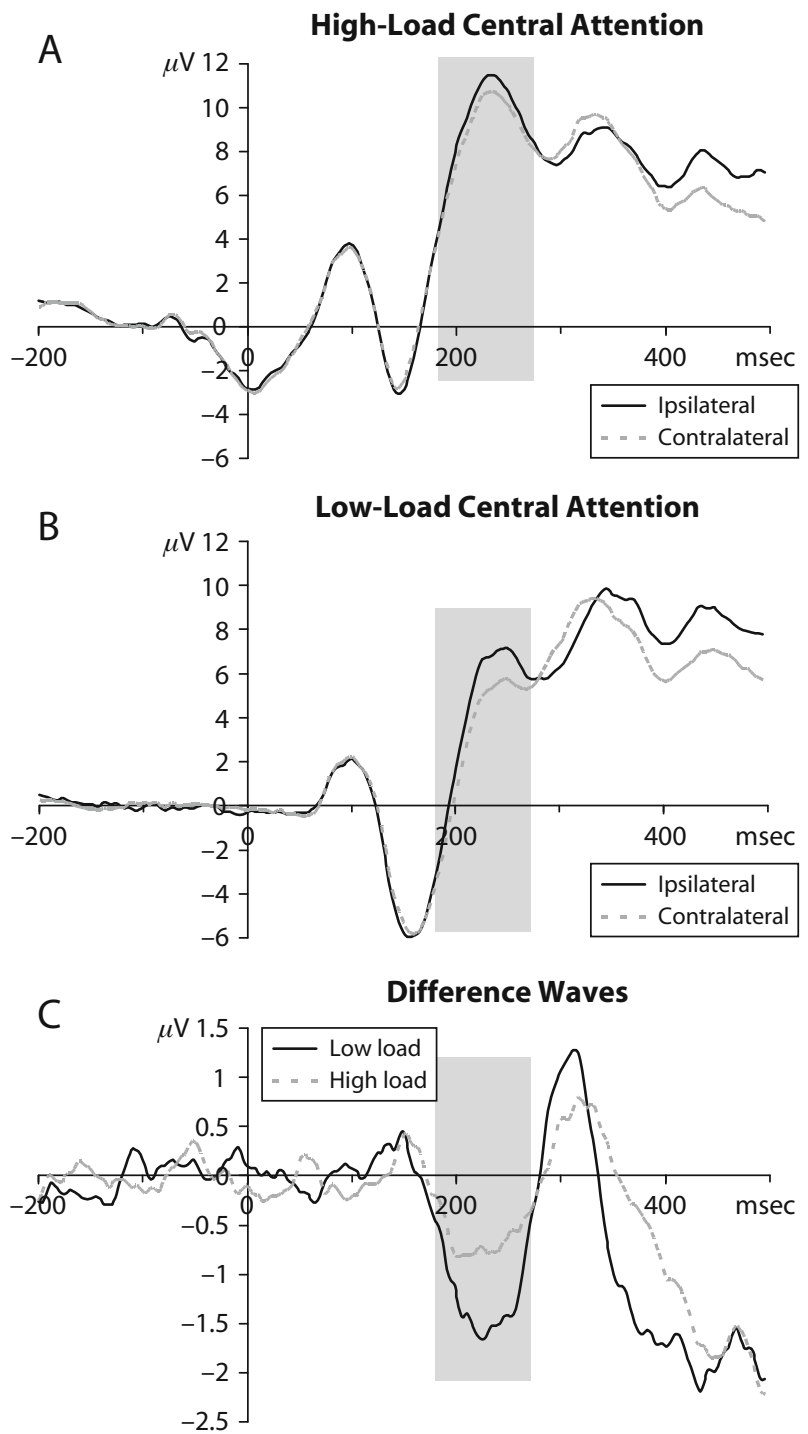

Figure 2. Electrophysiological results from Experiment 1. Grand average ipsilateral and contralateral event-related potential waveforms for (A) the high-load central attention condition and $(B)$ the low-load central attention condition time locked to the second target at lateral occipital PO7/PO8 sites (where the N2pc was maximal). (C) Difference waves (contralateral - ipsilateral waveforms) for both conditions.

$111.4, p<.0001],{ }^{5}$ which demonstrates that we obtained the desired PRP effect. No $\mathrm{T}_{2}$ accuracy effect was found $[F(1,7)<1]$, probably because performance approached ceiling.

Ipsilateral and contralateral waveforms at PO7/PO8 sites, as well as the difference waves for both conditions, are shown in Figure 4. As in Experiment 1, there was no electrode $(\mathrm{O} 1 / \mathrm{O} 2$ vs. $\mathrm{PO} 7 / \mathrm{PO} 8$ vs. $\mathrm{P} 7 / \mathrm{P} 8) \times$ central load (4AD vs. SRT) interaction. Furthermore, the N2pc was completely abolished in the 4AD condition $(t=$ $-1.30, p>.23)$, resulting in a main effect of central load $[F(1,7)=9.6, p<.018]$. This result replicates the N2pc results of Experiment 1, although stimuli were identical in both central load conditions.
It is interesting to note that $\mathrm{T}_{2}$ accuracy was not affected

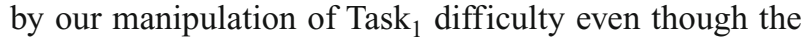
$\mathrm{N} 2 \mathrm{pc}$ was abolished in the 4AD condition. This result suggests that visuospatial attention may not be crucial for accurate performance in identifying highly overlearned stimuli presented in a visual display with a minimal number of distractors. It is likely that stronger effects would be observed for more complex tasks and/or in the presence of a higher level of competition for processing resources, as was seen in Experiment 1 (see, e.g., Awh, Matsukura, $\&$ Serences, 2003). We suppose that N2pc is generated by reentrant processing designed to enhance the signalto-noise ratio of the target and that the bottom-up signals, not enhanced by the processes generating the N2pc, were sufficient to achieve a high level of performance in the present tasks.

\section{GENERAL DISCUSSION}

The amplitude of the N2pc was sharply attenuated when the subjects attempted to deploy visuospatial attention while performing a capacity-demanding speeded auditory choice task. The observed N2pc reduction was not caused by a failure of color perception per se, which is essential to locate the color-defined target toward which visuospatial attention must be deployed. Indeed, a separate behavioral control experiment was conducted, in which we compared the central load effects in a condition identical to that of Experiment 1 (gap condition) with the central load effects in a condition with identical stimuli and an identical first task, but for which Task $_{2}$ required a four-alternative discrimination of the location of the uniquely colored $\mathrm{T}_{2}$ in the visual display (location condition). A central load effect in $\mathrm{T}_{2}$ accuracy in the gap condition but not in the location condition ${ }^{6}$ indicated that the subjects could locate the visual target as efficiently in the high-load as in the low-load condition, and therefore that the N2pc reduction observed in this study could not be explained by a failure of color perception in the high-load condition.

The present work is the first demonstration of interference with the N2pc response by concurrent central processing in the context of the PRP paradigm. We assume

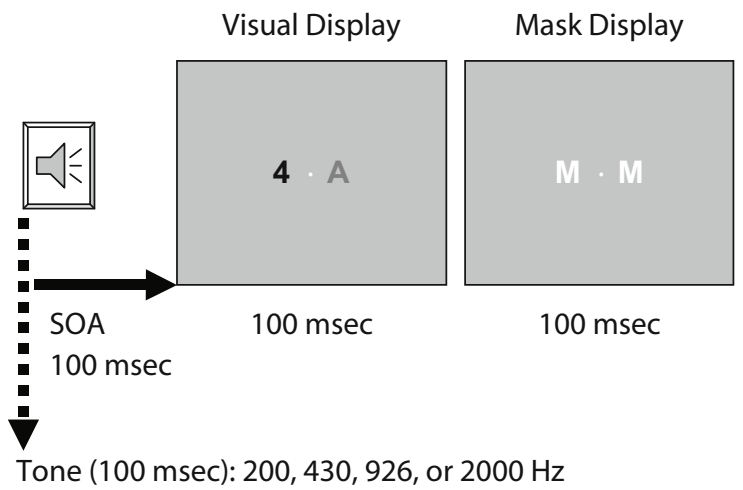

Figure 3. Stimulus sequence in Experiment 2. In the experiment, the items were red and green in the visual display and gray in the mask display. 

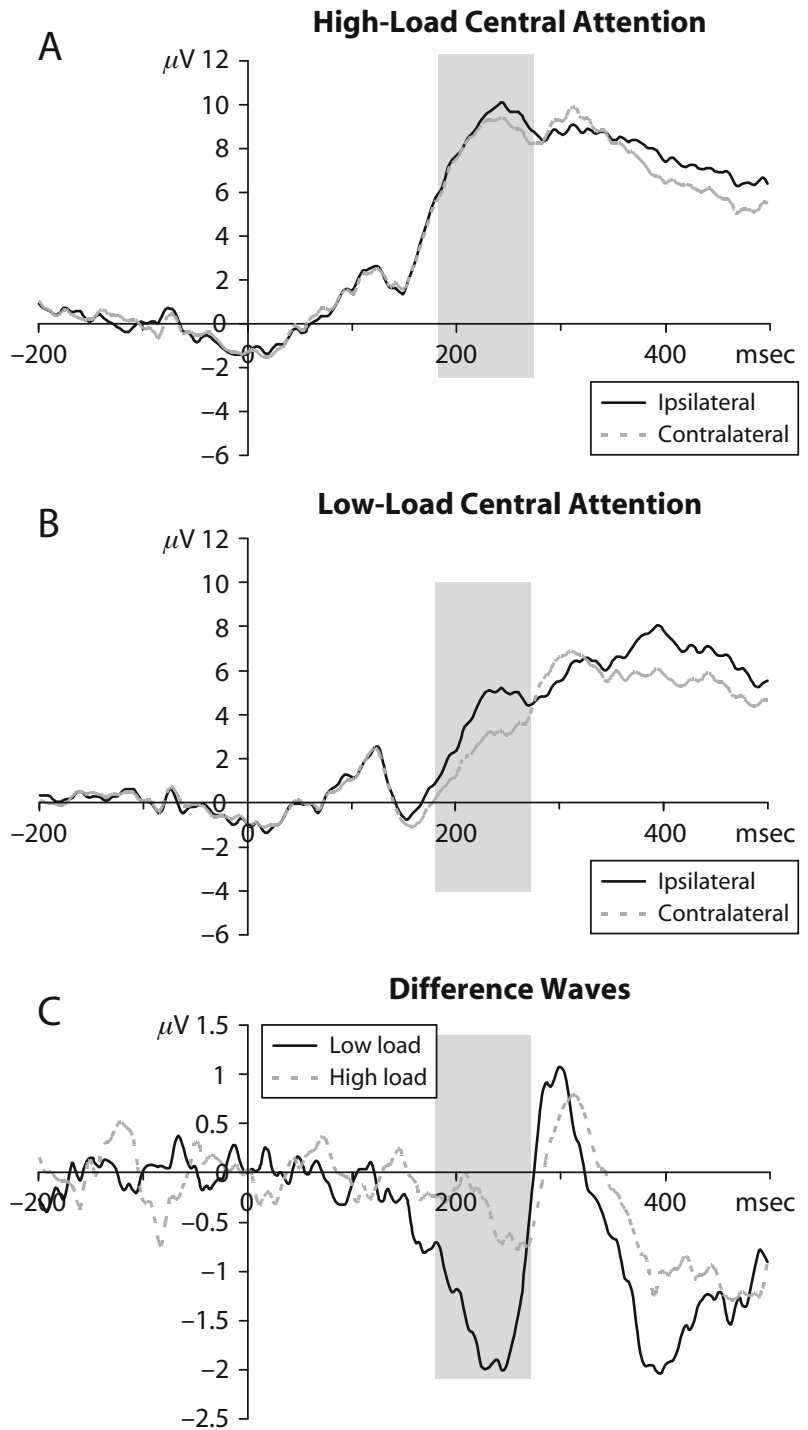

Figure 4. Electrophysiological results from Experiment 2. Grand average ipsilateral and contralateral event-related potential waveforms for (A) the high-load central attention condition and (2) the low-load central attention condition time locked to the second target at lateral occipital PO7/PO8 sites (where the N2pc was maximal). (C) Difference waves (contralateral - ipsilateral waveforms) for both conditions.

that the N2pc reflects the successful deployment of attention to the lateralized visual target (Eimer, 1996; Luck \& Hillyard, 1994; Woodman \& Luck, 2003). The attenuation of N2pc caused by concurrent central processing suggests that the deployment of visuospatial attention, or the control of this process, suffered significant central interference.

The results of the present study extend and provide converging support for related findings using the attentional blink (AB) paradigm (Jolicœur, Sessa, Dell'Acqua, \& Robitaille, 2006a, 2006b). In the AB paradigm, accuracy of report for some aspect of a masked $\mathrm{T}_{2}$, such as target identity, suffers when $\mathrm{T}_{2}$ is presented at a short SOA following a $\mathrm{T}_{1}$ that must also be processed. Jolicœur et al. (2006a, 2006b) used $\mathrm{T}_{2}$ displays similar to the ones used in the present work following presentation of another visual stimulus $\left(T_{1}\right)$ that did not require an immediate response. The N2pc was sharply attenuated by the AB. In these AB experiments, however, evidence for visual capture of attention (see, e.g., Folk, Leber, \& Egeth, 2002) was clearly apparent in some of the results (Jolicœur et al., 2006a) and could not be ruled out completely in others (Jolicœur et al., $2006 \mathrm{~b}$ ) - that is, processing of $T_{1}$ appeared sometimes to be associated with visual capture at the location of $\mathrm{T}_{1}$.

The present results cannot reflect visual capture because the first target in the PRP experiments was an auditory stimulus. We also do not believe that a form of crossmodal spatial capture (see McDonald \& Ward, 2000) associated with the location of the source of the sound is likely. The tones, presented with a pair of speakers behind the monitor, did not appear to come from a well localized point in space, but rather filled a large volume in the room. In any case, the present results provide clear-cut evidence for the involvement of central attention, because different degrees of N2pc attenuation were observed for identical stimuli associated with different tasks (Experiment 2). Any spatial capture associated with the onset of $\mathrm{T}_{1}$ would be the same for these stimuli. Moreover, the differential attenuation of the $\mathrm{N} 2 \mathrm{pc}$ was also observed in the absence of any possible differential pretrial preparatory state (Experiment 1). The N2pc modulation across conditions had to be due to the different concurrent central processing demands in Task ${ }_{1}$.

One could argue that the observed central load interference on the N2pc in the present study could reflect interference in task preparation after trial initiation, because the two SOAs used in Experiment 1 were very different. The interference-in-task-preparation hypothesis would state that, because the subjects were preparing for Task ${ }_{1}$, they could not set their "color filter" as efficiently in the short-SOA condition as in the long-SOA condition. As a consequence, on a portion of trials visuospatial attention would have been deployed to a distractor item opposite the target or not at all. Consequently, an attenuation of the difference in lateralized attention-related activity (i.e., the N2pc) in the averaged ERPs would be predicted. Although this argument does not contradict our claim that concurrent central processing interfered with the control of visuospatial attention because optimal preparation for Task $_{2}$ could not be maintained concurrently with the processing required for Task ${ }_{1}$, it does imply a different kind of interference than the bottleneck or capacity sharing that is postulated to be responsible for the behavioral PRP effect. The present results cannot falsify this task preparation hypothesis, and more work will be required to determine whether the interference we observed in the present work arose because of central postponement (or capacity sharing) or because of task preparation. However, a follow-up study (Brisson \& Jolicœur, 2007), which produced a stepwise attenuation of N2pc amplitude across SOAs of 1,000, 650 , and $300 \mathrm{msec}$, suggests that $\mathrm{N} 2 \mathrm{pc}$ attenuation can be found under conditions that make differential preparation very unlikely.

Although Pashler (1991), using a paradigm very similar to the one used here, concluded that central attention 
and visuospatial attention were independent, it is important to note that he nevertheless observed a significant reduction in $\mathrm{T}_{2}$ accuracy between the shortest and longest SOAs when color was used as the selection index $(4.7 \%$, $p<.005)$ and when attention had to be deployed to the opposite side of a peripheral onset cue $(5.1 \%, p<.001$; see also Jolicœur, 1999b, and Jolicœur \& Dell'Acqua, 1999, for similar SOA effects on $\mathrm{T}_{2}$ accuracy when $\mathrm{T}_{2}$ was masked in variants of the PRP paradigm). Several of the experiments reported by Pashler (1991) also showed evidence of interference in the form of long response times and/or decreased accuracy in Task ${ }_{1}$ at short SOAs. Note that Pashler (1991) used two-alternative discrimination in $\mathrm{Task}_{1}$, whereas we used more demanding four-alternative discriminations, likely making our results more systematic (see Dell'Acqua \& Jolicœur, 2000). The present electrophysiological results allow us to interpret these consistent SOA effects as evidence of central interference on the deployment of visuospatial attention.

Our results may appear to contradict earlier electrophysiological and behavioral results suggesting that stimuli could be processed deep in the cognitive system (at the level of meaning) without interference from bottlenecks in central processing (see, e.g., Vogel, Luck, \& Shapiro, 1998). This contradiction is more apparent than real, however, because in all previous electrophysiological work on the attentional blink, with the exception of Jolicœur et al. (2006a, 2006b), stimuli were presented at fixation and thus the impact of central load on the deployment of visuospatial attention could not be assessed.

The similarity of the interaction of central load on the $\mathrm{N} 2 \mathrm{pc}$ mean amplitude when PRP and AB paradigms are used provides more evidence in support of the central interference theory (Jolicœur, 1998, 1999a), which postulates that response selection (hypothesized to be an important locus of the PRP effect) and short-term consolidation (hypothesized to be an important locus of the $\mathrm{AB}$ effect) have some overlap at the level of limited central mechanisms. Although our results provide clear-cut demonstrations of the interactions between central load and N2pc amplitude, further work will be required to understand these interactions in more detail. For example, at the moment we do not know whether we interfered with the displacement of the attentional locus per se, or the interference was further downstream (e.g., in a failure to engage at the new location). Nonetheless, our new methods provide powerful tools to investigate the neural and psychological mechanisms that underlie the control of visuospatial attention.

\section{AUTHOR NOTE}

This research was supported by grants awarded to P. J. from the Natural Sciences and Engineering Research Council of Canada (NSERC), the Canada Research Chairs Program, and the Université de Montréal, and by an NSERC postgraduate scholarship awarded to B.B. Correspondence concerning this article should be addressed to B. Brisson, Département de Psychologie, Université de Montréal, C.P. 6128, succursale Centre-ville, Montréal, QC, H3C 3J7 Canada (e-mail: benoit.brisson@umontreal.ca).

\section{REFERENCES}

Awh, E., Matsukura, M., \& Serences, J. T. (2003). Top-down control over biased competition during covert spatial orienting. Journal of Experimental Psychology: Human Perception \& Performance, 29, 52-63.

Brisson, B., \& Joliceur, P. (2007). A psychological refractory period in access to visual short-term memory and the deployment of visual-spatial attention: Multitasking processing deficits revealed by event-related potentials. Psychophysiology, 44, 323-333.

Dell'ACQua, R., \& Jolicceur, P. (2000). Visual encoding of patterns is subject to dual-task interference. Memory \& Cognition, 28, 184-191.

EIMER, M. (1996). The N2pc component as an indicator of attentional selectivity. Electroencephalography \& Clinical Neurophysiology, 99, 225-234.

FolK, C. L., Leber, A. B., \& Egeth, H. E. (2002). Made you blink! Contingent attentional capture produces a spatial blink. Perception \& Psychophysics, 64, 741-753.

JiANG, Y., \& ChUN, M. M. (2001). The influence of temporal selection on spatial selection and distractor interference: An attentional blink study. Journal of Experimental Psychology: Human Perception \& Performance, 27, 664-679.

Johnston, J. C., McCann, R. S., \& Remington, R. W. (1995). Chronometric evidence for two types of attention. Psychological Science, 6, 365-369.

JoLICCEUR, P. (1998). Modulation of the attentional blink by on-line response selection: Evidence from speeded and unspeeded Task deci- $_{1}$ sions. Memory \& Cognition, 26, 1014-1032.

JoliCEUR, P. (1999a). Concurrent response-selection demands modulate the attentional blink. Journal of Experimental Psychology: Human Perception \& Performance, 25, 1097-1113.

Jolicceur, P. (1999b). Dual-task interference and visual encoding. Journal of Experimental Psychology: Human Perception \& Performance, 25, 596-616.

Joliceeur, P., \& DelL'ACQuA, R. (1999). Attentional and structural constraints on visual encoding. Psychological Research/Psychologische Forschung, 62, 154-164.

Joliceur, P., Sessa, P., Dell’Acqua, R., \& Robitaille, N. (2006a). Attentional control and capture in the attentional blink paradigm: Evidence from human electrophysiology. European Journal of Cognitive Psychology, 18, 560-578.

Joliceur, P., Sessa, P., Dell'Acqua, R., \& Robitaille, N. (2006b). On the control of visual spatial attention: Evidence from human electrophysiology. Psychological Research, 70, 414-424.

Karlin, L., \& Kestenbaum, R. (1968). Effects of number of alternatives on the psychological refractory period. Quarterly Journal of Experimental Psychology, 20, 167-178.

LuCK, S. J., \& HiLlyard, S. A. (1994). Spatial filtering during visual search: Evidence from human electrophysiology. Journal of Experimental Psychology: Human Perception \& Performance, 20, 1000-1014.

Mangun, G. R. (1995). Neural mechanisms of visual selective attention. Psychophysiology, 32, 4-18.

MCDONALD, J. J., \& WARD, L. M. (2000). Involuntary listening aids seeing: Evidence from human electrophysiology. Psychological Science, 11, 167-171.

Pashler, H. (1991). Shifting visual attention and selecting motor responses: Distinct attentional mechanisms. Journal of Experimental Psychology: Human Perception \& Performance, 17, 1023-1040.

PAshler, H. (1994). Dual-task interference in simple tasks: Data and theory. Psychological Bulletin, 116, 220-244.

Pashler, H., \& Johnston, J. C. (1989). Chronometric evidence for central postponement in temporally overlapping tasks. Quarterly Journal of Experimental Psychology, 41A, 19-45.

Posner, M. I. (1980). Orienting of attention. Quarterly Journal of Experimental Psychology, 32, 3-25.

Treisman, A., \& Gelade, G. (1980). A feature-integration theory of attention. Cognitive Psychology, 12, 97-136.

Vogel, E. K., LUCK, S. J., \& SHAPIRO, K. L. (1998). Electrophysiological evidence for a postperceptual locus of suppression during the at- 
tentional blink. Journal of Experimental Psychology: Human Perception \& Performance, 24, 1656-1674.

Woodman, G. F., \& Luck, S. J. (2003). Serial deployment of attention during visual search. Journal of Experimental Psychology: Human Perception \& Performance, 29, 121-138.

\section{NOTES}

1. Inclusion of subject(s) with no N2pc in the analyses did not change the pattern of results (i.e., it did not render significant effects nonsignificant or vice versa).

2. One subject grouped his responses and therefore presents a pattern of $\mathrm{RT}_{1}$ that is quite different from those of the other subjects. We therefore excluded this subject from $\mathrm{RT}_{1}$ analyses. However, it is known that grouping does not influence Task ${ }_{2}$ performance (see Pashler \& Johnston, 1989). Therefore, this subject was included in the other analyses.

3. ANOVAs in which target side (left vs. right) was included as an additional factor revealed no main effect of target side $\left[M_{\text {left }}=1,043\right.$, $\left.M_{\text {right }}=1,062 ; F(1,7)=1.27, p>.30\right]$ and no interaction of target side with SOA $[F(1,7)=1.46, p>.27]$.

4. One might wonder whether or not the N2pc amplitude attenuation could have been the result of jitter in the onset of the deployment of visuospatial attention in the short-SOA condition. Indeed, an all-or-none bottleneck model would predict that if central attention interfered with visuospatial attention, then the deployment of visuospatial attention to $T_{2}$ would occur only after the response to $T_{1}$ has been selected. This kind of interference would result in a latency jitter of $\mathrm{N} 2 \mathrm{pc}$ onset relative to $\mathrm{T}_{2}$ onset. However, according to the all-or-none bottleneck model, the jitter in the onset of the deployment of attention to $\mathrm{T}_{2}$ should be minimized relative to time of the response to $\mathrm{T}_{1}$. Therefore, if the $\mathrm{N} 2 \mathrm{pc}$ attenuation observed here is the result of jitter, then the N2pc should be restored in the short-SOA condition when the ERPs are time locked to the $\mathrm{T}_{1}$ response. N2pc-like difference waveforms time locked to $T_{1}$ response were computed. Contrary to the jitter hypothesis, these N2pc-like difference waves were completely flat in the high-load condition in both Experiment 1 and Experiment 2, providing no support for the hypothesis that the attenuation of $\mathrm{N} 2 \mathrm{pc}$ was due to component jitter.

5. ANOVAs in which target side (left vs. right) was included as an additional factor revealed no main effect of target side $[F(1,7)<1]$ and no interaction of target side with central load $[F(1,7)=1.8, p>.22]$.

6. $\mathrm{T}_{2}$ accuracy for the gap condition was lower in the short-SOA condition $\left[M_{\text {high load }}=72 \%, M_{\text {low load }}=82 \% ; F(1,7)=27.4, p<.002\right] . \mathrm{T}_{2}$ accuracy did not vary across load in the location condition $\left(M_{\text {high load }}=\right.$ $\left.91 \%, M_{\text {low load }}=94 \% ; F(1,8)=3.19, p>.11\right]$. These results produced a two-way interaction between Task $_{2}$ condition and SOA $[F(1,7)=9.2$, $p<.02]$.

(Manuscript received July 6, 2005; revision accepted for publication May 13, 2006.) 